\title{
Dielectric Resonators for the Measurements of the Surface Impedance of Superconducting Films
}

\author{
N. Pompeo, K. Torokhtii, and E. Silva \\ Dipartimento di Ingegneria, Università Roma Tre, Via della Vasca Navale 84, 00146 Roma, Italy, \\ enrico.silva@uniroma3.it
}

\begin{abstract}
We present the development, realization and setup of dielectric resonators, for the purpose of measuring the surface impedance at microwave frequencies of superconducting thin films. We focus on resonators designed to operate in de magnetic fields, optimized for the measurements of the variation of the surface impedance with the applied field. Two resonators, operating at 8 and 48 GHz, are presented. We discuss different approaches to the measurement of the resonator parameters, with particular attention to the nonidealities of real setups in a cryogenic environment. Finally, we present some sample measurement of high- $T_{c}$ and low- $T_{c}$ superconducting films.
\end{abstract}

Keywords: Microwave methods, dielectric resonators, superconductors, surface impedance, thin films, low temperature.

\section{INTRODUCTION}

$\mathrm{S}$ INCE the early times [1], the characterization at microwave frequencies of superconducting materials has been a topic of diffuse interest, due to its fundamental as well as technological importance [2,3]. Aim of this paper is to contribute to this topic, by presenting two dielectric resonators operating at low temperature and in high magnetic field.

The physical quantity which is experimentally accessible is the surface impedance $Z_{S}=R_{s}+\mathrm{i} X_{s}=E_{\|} / h_{\|}$[4]. In the local limit, one has $Z_{s}=Z_{s, \text { bulk }}=\left(\mathrm{i} 2 \pi v \mu_{0} \tilde{\rho}\right)^{1 / 2}$, where $\tilde{\rho}$ is the complex resistivity and $v$ is the measuring frequency. When the thickness $t_{s}$ of the sample under study is smaller than the London penetration depth (in the superconducting state) or the skin depth (in the normal state), one has the important approximate relation [5]:

$$
Z_{s} \simeq Z_{s, f i l m}=\frac{\tilde{\rho}}{t_{s}}
$$

Thus, in sufficiently thin samples one has direct access to the complex resistivity, a material parameter of paramount importance in the characterization of superconductors.

Many experimental techniques have been developed for the study of superconductors [6]. In general, resonant techniques are more accurate than nonresonant methods $[7,8,9,10,11$, $12,13]$. While the ultimate sensitivity is obtained with superconducting cavities [14], this method is definitely not feasible when the sample has to be characterized under a dc magnetic field. In this case, metallic cavities [15] or dielectric resonators in low-indices $[16,17,18,19]$ as well as in whispering gallery modes $[20,21,22,23]$ can be used. Dielectric resonators exhibit increased sensitivity, and more compact dimensions. Their advantage resides in the capability of measuring unpatterned thin films, using the so called surface perturbation method $[24,25]$ : the sample substitutes part of a metallic wall, thus contributing to dissipation (resistance) and energy storage (reactance) in the resonator. By measuring the quality factor $Q$ and resonant frequency $v_{0}$ of the resonator $[17,18,19,20]$ one can derive $Z_{s}$. The materials and the geometry chosen for the practical realization of the resonator, as well as the methods adopted for the determination of $Q$ and $v_{0}$, affect the sensitivity, the accuracy and the precision of the measurements of $Z_{S}$.

For what concerns the geometry of the resonator, we concentrate on dielectric-loaded cylindrical resonators, based on a cylindrical dielectric rod, with complex permittivity $\varepsilon_{r}=$ $\varepsilon_{r}^{\prime}\left(1+\mathrm{i} \tan \delta_{\varepsilon}\right)$, screened by a metallic enclosure of surface impedance $Z_{m}$. Excitation and detection of the signal is obtained by loops or antennas, which introduce additional losses. Thus, the assembly exhibits a loaded quality factor $Q_{L} \neq Q$. With the typical transmission configuration, one has, $Q=Q_{L}\left(1+\beta_{1}+\beta_{2}\right)$, where $\beta_{1,2}<1$ are the coupling coefficients. In practice, depending on the setup it may prove useful to reduce coupling to a minimum, in order to approximate $Q_{L} \simeq Q$, or to separately calculate or measure $\beta_{1,2}$.

$Q$ and $v_{0}$ are related to $Z_{S}$ by the equations [19]:

$$
\begin{array}{r}
\frac{1}{Q}=\frac{R_{s}}{G_{s}}+\frac{R_{m}}{G_{m}}+\eta \tan \delta_{\varepsilon} \\
-2 \frac{\Delta v_{0}}{v_{0}}=\frac{\Delta X_{s}}{G_{s}}+\frac{\Delta X_{m}}{G_{m}}+\eta \frac{\Delta \varepsilon_{r}^{\prime}}{\varepsilon_{r}^{\prime}}
\end{array}
$$

where $\Delta A=A(x)-A\left(x_{\text {ref }}\right)$ is the variation of the quantity $A$ with respect to some reference value $A\left(x_{\text {ref }}\right)$ when a physical parameter $x$ is varied (e.g., the temperature $T$ or the external dc magnetic field $H$ ). The geometrical factors are related to the various surfaces of the resonator $\left(G_{s, m}\right)$ [4] or to the ratio of the energy stored in the dielectric and in the empty space of the resonator ( $\eta$, with $\eta \approx 1$ ). One should note that no absolute measurement of $X_{S}$ is possible, unless a reference value is either assumed or separately measured or derived.

The measurement of $Z_{s}$ in a cryogenic environment presents some typical difficulties. In particular, the contribution of the transmission lines cannot be easily calibrated: it 
is almost impossible to replicate the thermal profile along the line in different measurement runs. Moreover, the low losses of superconductors make the choice of the dielectric critical.

We discuss in Sec. 2 the main factors affecting the quality of the measurements, and in Sec. 3 the results of the practically obtained resonators, before concluding in Sec.4.

\section{Methods}

In this Section we exploit the various quality indicators of the measurements, for a proper choice of the materials of the resonators.

Sensitivity, accuracy - We focus on the measurement of $R_{S}$. However, most of the arguments apply also to $\Delta X_{s}$ : Equations (2) and (3) show that the same geometrical factors are involved, in a very similar way. From (2) and (3) one finds the sensitivity and accuracy for $R_{S}$ :

$$
\begin{aligned}
\frac{\partial Q}{\partial R_{s}}= & -\frac{1}{G_{s}} Q^{2} \\
\left|\frac{\delta R_{s}}{R_{s}}\right|= & {\left[\left(1+\frac{G_{s} R_{m}}{R_{s} G_{m}}+\frac{G_{s}}{R_{s}} \eta \tan \delta_{\varepsilon}\right)^{2}\left|\frac{\delta Q}{Q}\right|^{2}\right.} \\
& +\left|\frac{\delta G_{s}}{G_{s}}\right|^{2}+\left(\frac{G_{s} R_{m}}{R_{s} G_{m}}\right)^{2}\left(\left|\frac{\delta R_{m}}{R_{m}}\right|^{2}+\left|\frac{\delta G_{m}}{G_{m}}\right|^{2}\right) \\
& \left.+\left(\frac{G_{s}}{R_{s}} \eta \tan \delta_{\varepsilon}\right)^{2}\left(\left|\frac{\delta \eta}{\eta}\right|^{2}+\left|\frac{\delta \tan \delta_{\varepsilon}}{\tan \delta_{\varepsilon}}\right|^{2}\right)\right]^{\frac{1}{2}}
\end{aligned}
$$

Thus, to maximize the sensitivity one needs high $Q$ and small $G_{s}$. By an electromagnetic analysis, one finds that the sample must be placed where microwave currents are at their maximum.

Taking into account that the uncertainty on the loss tangent is usually large ( $\left|\delta \tan \delta_{\varepsilon} / \tan \delta_{\varepsilon}\right| \gtrsim 10 \%$ [20]), (5) requires the use of very low loss dielectrics, together with low $R_{m}$ metals for the resonator.

One should note that the uncertainty in the geometrical factors $G_{s, m}$ and $\eta$ do not add to the random error. Instead, they contribute to a systematic error. We will see that in fielddependent measurement such uncertainty plays a very minor role. By contrast, an accurate determination of $Q$ is mandatory, since it is the main source of uncertainty in the determination of $R_{s}$.

Repeatibility, precision - Operation in a cryogenic environment requires that the assembly must compensate the thermal stresses and thermal compression and expansion. Thus, appropriate springs, elastic from below $4 \mathrm{~K}$ up to room temperature, must be used. Since operation in a magnetic field (up to $10 \mathrm{~T}$ ) is required, Beryllium or phosphor bronze are typical materials. The frequency stability of the excitation is another critical point. Appropriate thermalization of the source (over several hours) can bring the frequency stability to the expected values $\left(10^{-3} \mathrm{ppm}\right.$ in a typical research-grade Vector Network Analyzer - VNA). This point is crucial for accurate measurements of $\Delta X_{s}$ (equation (3)).
Calibration of the resonator - The contribution of the metal and dielectric parts of the resonator to the overall $Q$ and $\Delta v_{0}$ can be subtracted with the following procedure.

First, at the temperatures of interest $Q_{o m g}$ and $\Delta v_{0, \text { omg }} / v_{0, \text { omg }}$ are measured, with the sample substituted by a metal piece of the same material than the body of the cavity (same $Z_{m}$ ). This configuration is called "homogeneous configuration". One then gets, from (2) and (3):

$$
\begin{array}{r}
\frac{1}{Q_{o m g}}=R_{m}\left(\frac{1}{G_{s}}+\frac{1}{G_{m}}\right)+\eta \tan \delta_{\varepsilon} \\
-2 \frac{\Delta v_{0, o m g}}{v_{0, o m g}}=\Delta X_{m}\left(\frac{1}{G_{s}}+\frac{1}{G_{m}}\right)+\eta \frac{\Delta \varepsilon_{r}^{\prime}}{\varepsilon_{r}^{\prime}}
\end{array}
$$

With a very low loss dielectric, $\eta \tan \delta_{\varepsilon}$ can be neglected in (2) and (6) and $R_{m}$ can be derived, as a function of $T$, from (6) (otherwise, independent measurements of $\tan \delta_{\varepsilon}$ are required $[20,26,27])$. However, in general in $\Delta X_{s}$ the contribution of the dielectric cannot be neglected, since $\varepsilon_{r}^{\prime}$ changes appreciably with $T$. This drawback can be circumvented by assuming $\Delta X_{m}=\Delta R_{m}$ (Hagen-Rubens limit, usually fulfilled in $\mathrm{Cu}$ at microwave frequencies) in (7).

When the subject of interest are the variations with the static magnetic field $H$ at fixed $T, Z_{S}(H, T)-Z_{S}(0, T)$, no calibration is needed and the accuracy improves noticeably. From (2) and (3) one gets:

$$
\begin{aligned}
R_{S}(H, T)-R_{S}(0, T) & =G_{S}\left(\frac{1}{Q(H, T)}-\frac{1}{Q(H, 0)}\right) \\
X_{S}(H, T)-X_{S}(0, T) & =-2 G_{S} \frac{v_{0}(H, T)-v_{0}(H, 0)}{v_{0}(H, 0)}
\end{aligned}
$$

Measurement of the response of the resonator - $\mathrm{A}$ transmission-operated resonator can be characterized by measuring the scattering coefficient $S_{21}(v)$, which in the ideal case reads $[28,29]$ :

$$
S_{21}(v)=\frac{S_{21}\left(v_{0}\right)}{1+2 \mathrm{i} Q_{L} \frac{v-v_{0}}{v_{0}}}
$$

where $S_{21}\left(v_{0}\right)=2 \sqrt{\beta_{1} \beta_{2}} /\left(1+\beta_{1}+\beta_{2}\right)$ (we neglect the effect of the coupling on the resonant frequency). Equation (10) can be written in canonical form as $S_{21}(v)=\left(a_{1} t+a_{2}\right) /\left(a_{3} t+1\right)$, with $t=\left(v-v_{0}\right) / v_{0}$.

$\left|S_{21}(v)\right|^{2}$ describes a lorentzian curve as a function of v. $S_{21}$ describes a circle in the complex plane $\left\{\operatorname{Re}\left(S_{21}\right)\right.$, $\left.\operatorname{Im}\left(S_{21}\right)\right\}$. From $S_{21}(v), Q$ and $v_{0}$ can be obtained by means of several methods [28, 29]. The measured transmission coefficient $S_{21, m}(v)$ includes all nonidealities of the transmission lines (cables, waveguides introduce phase delay and losses), of the couplers (losses, cross-talk), and noise, so that $S_{21, m}(v) \neq S_{21}(v)$. Moreover, in cryogenic measurements there is no way to calibrate precisely such effects. However, typical (good) transmission lines contribute mainly with a complex transmission coefficient $\alpha(v)$, so that one can write 
(a)

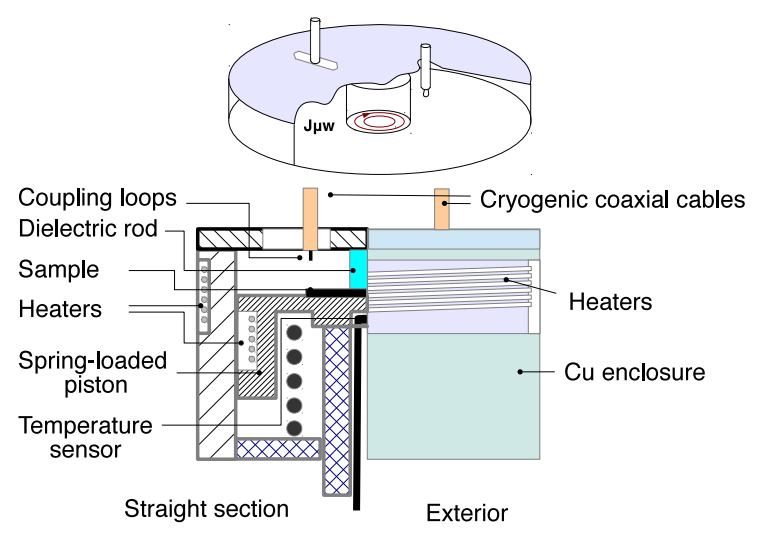

Fig. 1: (a) sketch of a typical dielectric resonator. Coupling loops are depicted, as realized in the $8 \mathrm{GHz}$ resonator. The circular geometry of the current density $\mathbf{J}$ on the sample is shown; (b) drawing of the 8 $\mathrm{GHz}$ resonator.

$S_{21, m}(v)=\alpha(v) S_{21}(v)$. This feature will prove useful in the fitting of the experimental data.

Since fitting is required in order to get the values for $Q$ and $v_{0}$, in the following we will give the uncertainty related to the fit in terms of the standard uncertainty, i.e. the square root of the diagonal elements of the covariance matrix of the fit parameters [30].

Design of the resonators - The Hakki-Coleman geometry [31] (Figure 1(a)) is used for the resonators here described. The dielectric rod is sandwiched between two conducting bases. The resonator operates in the $\mathrm{TE}_{011}$ mode, with circular, planar currents induced on the conducting bases. Planar currents are essential when investigating cuprate superconductors: their extreme anisotropy would introduce very significant uncertainties if some perpendicular current were present. This geometry is here chosen to measure single samples for research purposes. This choice requires that one of the bases remain of normal conductor, so that $G_{m} \sim G_{s}$ (the lateral walls contribute negligibly to the losses) ${ }^{1}$. One has typically $Q \sim 10^{4}$, with sensitivity $0.5-1 \mathrm{~m} \Omega$.

The request for reduction of the losses suggests the use of oxygen free high conductivity (OFHC) $\mathrm{Cu}$ for the metal enclosure, with [33] at $10 \mathrm{GHz}: R_{m}<15 \mathrm{~m} \Omega$ for $T<80 \mathrm{~K}$ and $R_{m}<1.5 \mathrm{~m} \Omega$ for $T<20 \mathrm{~K}$.

The request for very small $\tan \delta_{\varepsilon}$ requires the use of single crystals (differently from, e.g., telecommunication dielectric resonator filters [34]). Strong confinement of the electromagnetic field, which implies a reduction of the losses on the lateral wall and a more compact design, requires large $\varepsilon_{r}^{\prime}$. Thus, the choice of the dielectric is limited to sapphire, $\mathrm{Al}_{2} \mathrm{O}_{3}$, with $\varepsilon_{r}^{\prime} \simeq 9$ and $\tan \delta_{\varepsilon}<10^{-7}$ for $T<80 \mathrm{~K}$, and rutile, $\mathrm{TiO}_{2}$, with $\varepsilon_{r}^{\prime} \simeq 120$ and $\tan \delta_{\varepsilon}<10^{-6}$ for $T<20 \mathrm{~K}$ [35]. Thus, care must be taken in the use of rutile at temperatures above $20 \mathrm{~K}$. Both materials are uniaxially anisotropic (the value given for

${ }^{1}$ This geometry is used also to measure pairs of similar films place on both bases. One then has $G_{m} \rightarrow 0$, and the accuracy reaches values $\pm 30 \mu \Omega$ at $18 \mathrm{GHz}$ [32]. However, only the average response of the two samples is measured, that can be unacceptable in several cases.

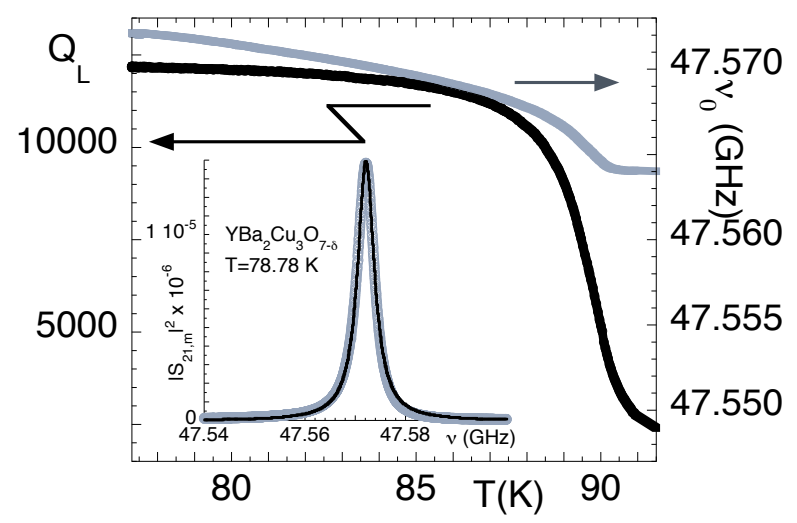

Fig. 2: Inset: frequency response of the $48 \mathrm{GHz}$ dielectric resonator operating in transmission. The experimental curve (gray line) is fitted to a lorentzian curve (black line), with parameters: $v_{0}=(47.572041 \pm 0.000001) \mathrm{GHz}, Q_{L}=11860 \pm 20$. Main panel: $Q_{L}(T)$ and $v_{0}(T)$ for a $\mathrm{YBa}_{2} \mathrm{Cu}_{3} \mathrm{O}_{7-x}$ film, showing the superconducting transition.

$\varepsilon_{r}^{\prime}$ refers to the plane perpendicular to the anisotropy axis, of interest here). At low temperature, $\tan \delta_{\varepsilon}$ strongly depends on impurities, so that each single crystal needs to be characterized.

In the following Section we describe the realization, the setup and operation of two resonators, operating at $48 \mathrm{GHz}$ and $8 \mathrm{GHz}$, respectively. Since both have typical values $Q \sim$ $10^{4}$ when the sample is superconducting, the uncertainty in the determination of $Q$ is $\sim 0.5-1 \%$. We also calculated in both cases $G_{s} \sim 10^{3}$, whence the sensitivity (4) $\delta R_{S} \sim 0.5-$ $1 \mathrm{~m} \Omega$. We note that below $Q \sim 10^{3}$ the sensitivity decreases noticeably, and reliable measurements are confined to $R_{s}^{\max } \lesssim$ $3 \Omega$ (three orders of magnitude dynamics). This is sufficient to describe most of the superconducting transition in films. Analogous sensitivity $\sim 1 \mathrm{~m} \Omega$ is obtained in the reactance, $\Delta X_{s}$. As a consequence, one needs frequency resolution $\lesssim$ $100 \mathrm{~Hz}$, within the capabilities of a typical VNA.

\section{RESUlts}

$48 \mathrm{GHz}$ resonator - The high operating frequency (to our knowledge, this is the dielectric resonator operating at the highest frequency for the characterization of superconductors) directly brings reduced dimensions. Thus, we could choose sapphire as a dielectric. The sapphire rod has dimensions: $h=(2.918 \pm 0.002) \mathrm{mm}$ (height), $D_{\text {diel }}=(1.748 \pm 0.004) \mathrm{mm}$ (diameter). The metal enclosure has a diameter $D_{\text {ris }}=9 \mathrm{~mm}$. The use of sapphire allows operation up to ambient temperature. The cryostat operates down to $50 \mathrm{~K}$.

This resonator is a modification of a reflection-mode resonator [19]. An important consequence of the transmission measurement resides in that very small coupling coefficient can be used: $\beta_{1,2} \lesssim 0.5 \%$. This allows, contrary to reflection measurements, to assume $Q \simeq Q_{L}$, and there is no need to measure the absolute value of $S_{21}$. A synthesized source and crystal detectors were used, and typical resonance curves were obtained from measurements of $\left|S_{21}\right|$ as reported in the 


\section{inset of Figure 2.}

From measurements of $Q$ and $v_{0}$ as a function of the temperature, one obtains several useful information. In fact, the microwave technique is powerful in the identification of spurious or additional superconducting phases: since the electromagnetic field penetrates in the full body of the superconductor (in thin films), additional superconducting phases would appear as more or less rounded "steplike" transitions, as opposed to dc measurements where the first appearance of a percolative superconducting path between the electrodes would completely mask the behaviour below that temperature.

A first example is reported in Figure 2: we show the microwave response of a thin $\mathrm{YBa}_{2} \mathrm{Cu}_{3} \mathrm{O}_{7-x}$, high- $T_{c}$ superconducting film, grown on a $\mathrm{SrTiO}_{3}$ substrate. From the temperature dependence of $Q$ and $v_{0}$ we identified the transition temperature $T_{c}=90.5 \mathrm{~K}$. The inspection of $Q(T)$ and $v_{0}(T)$ clearly shows a regular superconducting transition, with no traces of multiphase superconductivity. The second example concerns a $\mathrm{Tl}_{2} \mathrm{Ba}_{2} \mathrm{CaCu}_{2} \mathrm{O}_{8+x}$ thin film, grown on $\mathrm{CeO}_{2}$ buffered sapphire. From $Q(T)$ and $v_{0}(T)$ we identified the transition temperature $T_{c}=103.6 \mathrm{~K}$. Figure 3(a) clearly shows that our sample is single-phase. This sample presents a strong dissipation in a magnetic field, that can be used to exemplify the dynamic range of the present resonator. We made use of the fact that the penetration depth in $\mathrm{Tl}_{2} \mathrm{Ba}_{2} \mathrm{CaCu}_{2} \mathrm{O}_{8+x}$ is estimated to be $\sim 250 \mathrm{~nm}$. We then exploited (1) and (8) to derive the magnetic-field-induced increase of the real part of the complex resistivity. The increase of the dissipation is due to the motion of quantized vortices ("fluxons") [36]. The study of such structures is obviously of essential importance for the applications, and due to the peculiar physics of high- $T_{c}$ superconductors has given rise to a branch of the study of superconductors by itself ("Vortex matter" [37]). In Figure 3(b) and 3(c), it can be seen that the microwave technique here reported allows for the determination of changes in resistivity with resolution better than $0.02 \mu \Omega \mathrm{cm}$, with a dynamic range over three decades.

$8 \mathrm{GHz}$ resonator - This resonator has been explicitly designed to work at relatively low frequency in conventional superconductors. In fact, in a magnetic field a superconductor exhibits a characteristic frequency for the motion of fluxons, which is below $1 \mathrm{GHz}$ in conventional superconductors [38]. Thus, to have extensive information on the motion of fluxons one needs to operate at frequencies not too high.

As a consequence of the requirement of relatively low operating frequency, high-permittivity rutile has been chosen for the dielectric rod. Since we are dealing with conventional superconductors, the operating temperature is well below 20 $\mathrm{K}$, so that dielectric losses are not significant. The dimensions of the rod are: height $h=(2.73 \pm 0.01) \mathrm{mm}$, diameter $D_{\text {diel }}=(3.90 \pm 0.05) \mathrm{mm}$. The dielectric is enclosed by a $\mathrm{Cu}$ OFHC screen. Measurements down to $3 \mathrm{~K}$ and up to $10 \mathrm{~T}$ are feasible. For what concerns the field-sweeps measurements, we found that temperature stabilization within $1 \mathrm{mK}$ was required, in order to avoid drifts of the resonant frequency.

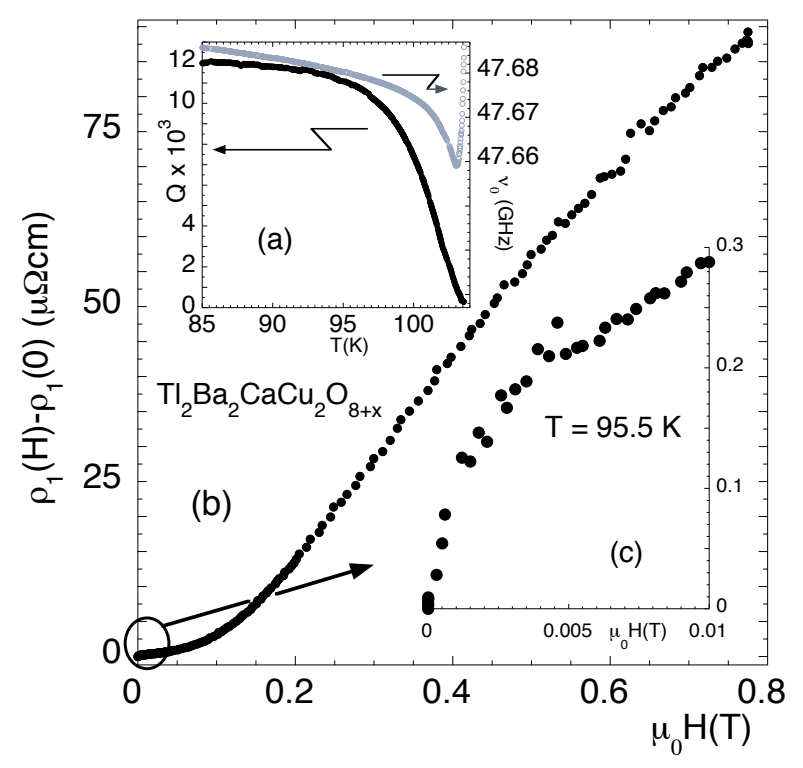

Fig. 3: (a) $Q$ and $v_{0}$ vs. $T$ for a thin $\left(t_{s}=240 \mathrm{~nm}\right) \mathrm{Tl}_{2} \mathrm{Ba}_{2} \mathrm{CaCu}_{2} \mathrm{O}_{8+x}$ film, measured at $48 \mathrm{GHz}$. (b) Magnetic-field-induced variation of $\operatorname{Re}(\tilde{\rho})$ at fixed $T$. The enlargement (c) allows to appreciate the sensitivity and the dynamic range.

A VNA (Anritsu) allowed measurements of $S_{21, m}$. As a consequence, we could derive $Q$ and $v_{0}$ both by means of lorentzian fits of $\left|S_{21, m}\right|$, analogously to the $48 \mathrm{GHz}$ resonator, and by fits of $S_{21, m}$ in the complex plane [28, 29]. Figure 4 shows an example of lorentzian fit, an example of ideal and nonideal resonance curves in the complex plane, and an example of experimental complex-plane circles, as raw data and after the data conditioning proposed in the following.

We note that the complex-plane fit is particularly suitable to compensate and reduce the contribution of nonidealities in $S_{21, m}$ : cross-talk and noise are easily removed by this kind of data analysis. In particular, cross-talk only shifts the center of the circle, leaving the estimate of the resonance parameters unaffected once this effect is taken into account. By contrast, the lorentzian curve may acquire a substantial asymmetry when cross-talk is present, up to the level that the fit is completely unreliable. However, the complex transmission coefficient of the line $\alpha$ can severely affect $S_{21, m}$. In particular, the phase delay can change the shape of $S_{21, m}$ in the complex plane. Since the phase delay depends on the temperature and on temperature gradients, reliable measurements are often obtained only after a long, time-consuming adjustment of the thermal conditions. Clearly, a removal of the effects of phase delay is desirable.

It has been proposed $[39,40]$ to exploit simultaneous measurements of the three complex scattering coefficients $S_{11, m}$, $S_{22, m}$ and $S_{21, m}$. This method requires appreciable coupling coefficients, and as such it can be useful with relatively large $\beta_{1,2}$. Uncertainties $<0.1 \%$ for $Q \geq 10^{6}$ are possible [41].

Seeking for a more general approach, we have developed and we describe in the following a procedure to remove the phase contribution in situ, irrespective of the coupling, when within reasonable limits. The attenuation given by the trans- 

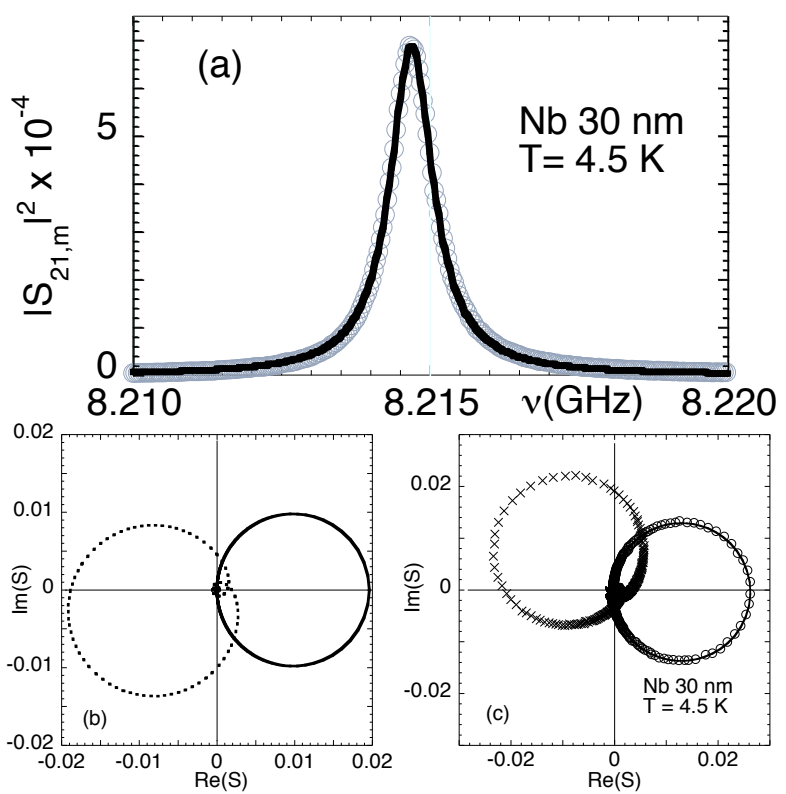

Fig. 4: (a) $\left|S_{21, m}\right|^{2}$ measured with the $8 \mathrm{GHz}$ resonator, and lorentzian fit, with parameters: $v_{0}=(8.2146855 \pm 0.0000003) \mathrm{GHz}$, $Q_{L}=9360 \pm 20$; (b) resonance circles in the complex plane $S_{21, m}$ calculated in the ideal case (continuous line), and with a phase delay (dashed line); parameters of the simulation: $Q=9000, v_{0}=8.2$ $\mathrm{GHz}$; (c) measurements of $S_{21, m}$ with the $8 \mathrm{GHz}$ resonator (crosses: raw data; open circles: data with compensation of the phase delay), and fit with the theoretical expression (continuous line), with parameters $Q_{L}=9370 \pm 20, v_{0}=(8.2146705 \pm 0.0000002) \mathrm{GHz}$.

mission lines, $|\alpha|$ (we neglect the frequency dependence: high $Q_{L}$ values imply narrow frequency ranges), affects only $S_{21}\left(v_{0}\right)$ (10), hence only the determination of $\beta_{1,2}$. While $|\alpha|$ has no effect on the determination of $Q_{L}$, the phase $\arg (\alpha)$ cannot be neglected and it may have a dramatic effect. As an example, in Figure 4(b) we have reported as a continuous line the ideal circle, calculated with (10), with parameters $S_{21}\left(v_{0}\right)=0.02, v_{0}=8.2 \mathrm{GHz}$ and $Q_{L}=9000$ and negligible coupling; to the same circle we added a phase contribution $\exp (\mathrm{i} \gamma v)$ (contribution of a transmission line), and the result is plotted as a dashed line. We concentrate here on the important role of the phase delay: as it can be seen, it completely distorts the response. The ideal circle undergoes to strong changes: it is rotated in the complex plane, and close to the origin it describes several characteristic "nodes". A blind fit would be very unreliable, as well as the addition of several parameters to take into account the nonidealities. We opted for a direct removal of the phase contribution from the raw data, as we show in the following.

Let $S_{21, m}$ be the measured scattering coefficient for a resonator as in Figure 4(a), with uncalibrated transmission lines. Far from the resonance, we experimentally verified $\arg \left(S_{21, m}\right) \propto v$, according to the theory of transmission lines [4]. Thus, we reconstruct $\arg (\alpha)=a+b v$ by measuring $\arg \left(S_{21, m}\right)$ far enough from $v_{0}$. We note that at $v_{0}, \arg \left(S_{21, m}\right)$ has a step. However, the removal of the transmission line phase must include only data where the phase is linear with
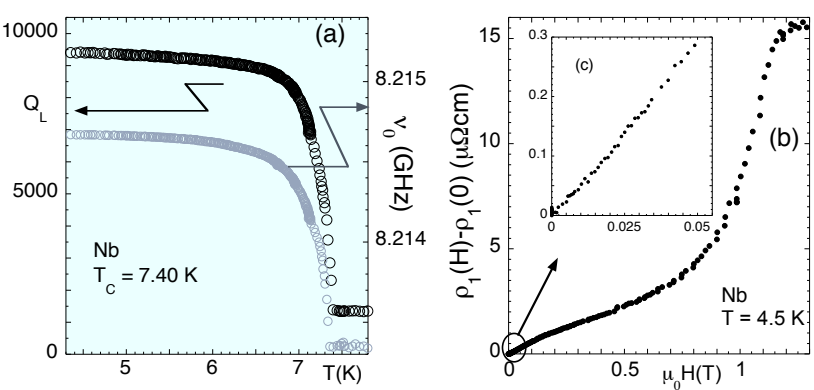

Fig. 5: (a) $Q$ and $v_{0}$ vs. $T$ for a thin $\left(t_{s}=30 \mathrm{~nm}\right) \mathrm{Nb}$ film, measured at $8 \mathrm{GHz}$. (b) Magnetic-field-induced variation of $\operatorname{Re}(\tilde{\rho})$ at fixed $T$. The enlargement (c) allows to appreciate the sensitivity and the dynamic range.

$v$. This is accomplished by evaluating the linear regression coefficient by progressively removing more data around $v_{0}$, until it stops changing. This procedure is adopted separately on both sides of $v_{0}$, with the constraint that the slope $b$ is the same. Once the spurious contribution is obtained, it can be subtracted from $S_{21, m}$. This procedure can be applied in real time, with obvious time saving in the measurements.

In Figure 4(c) we report a typical experimental measurement of $S_{21, m}$, taken at $4.70 \mathrm{~K}$ in the range $8.49-8.51 \mathrm{GHz}$ with the resonator loaded with a superconducting $\mathrm{Nb}$ film [42]. The raw data (crosses) clearly describe a misshaped, asymmetric circle, with spurious "nodes" in the origin, originating from the uncalibrated phase contribution. By applying the procedure described above the circle rotates and the "nodes" in the origin are removed, giving a well-shaped circle (open symbols). The fit (continuous line) is of good quality. Most important, the procedure allows for the direct identification and removal of the phase contributions to measuring errors. We conclude that the complex plane fit can be routinely performed, with a direct subtraction from the data of the phase contribution of uncalibrated lines.

In Figure 5 we report examples of measurements taken on a thin $\left(t_{s}=30 \mathrm{~nm}\right) \mathrm{Nb}$ film, similarly to the measurements reported at $48 \mathrm{GHz}$ in $\mathrm{Tl}_{2} \mathrm{Ba}_{2} \mathrm{CaCu}_{2} \mathrm{O}_{8+x}$. We obtain here $T_{c}=7.40 \mathrm{~K}$. This resonator exhibits (Figure $5 \mathrm{~b}$ ) a similar sensitivity to the $48 \mathrm{GHz}$ resonator, showing that at low temperatures rutile can be used safely instead of sapphire.

\section{CONCLUSIONS}

In this work we have briefly reviewed the application of the dielectric resonator technique to the measurements of the surface impedance in low loss, superconducting planar materials. We have presented the design and realization of two dielectric resonators, different for the frequency range and the dielectric properties. We have introduced the circle fit method, and we have shown that it allows to remove the important phase delay contribution to transmission measurements. Finally, we have demonstrated the operation of the resonators by presenting sample measurements in high- $T_{c}$ and conventional superconductors. 


\section{ACKNOWLEDGEMENT}

N.P. acknowledges support from Regione Lazio. We gratefully thank H. Schneidewind, G. Celentano and C. Attanasio for the $\mathrm{Tl}_{2} \mathrm{Ba}_{2} \mathrm{CaCu}_{2} \mathrm{O}_{8+x}, \mathrm{YBa}_{2} \mathrm{Cu}_{3} \mathrm{O}_{7-x}$ and $\mathrm{Nb}$ samples, respectively.

\section{REFERENCES}

[1] Oates, D. E. (2012). Microwave measurements of fundamental properties of superconductors. In 100 Years of Superconductivity. CRC Press, 459-471.

[2] Hein, M. (2010). High-Temperature Superconductor Thin Films at Microwave Frequencies, Springer.

[3] Weinstock, H., Nisenoff, M. (Eds.) (2001). Microwave Superconductivity. Kluwer Academic Publishers.

[4] Collin, R. E. (1992). Foundation for Microwave Engineering, 2nd Edition. McGraw-Hill.

[5] Silva, E., Lanucara, M., Marcon, R. The effective surface resistance of superconductor/dielectric/metal structures. (1996). Superconductor Science and Technology, 9 (11), 934-941.

[6] Maeda, A., Kitano, H., Inoue, R. (2005). Microwave conductivities of high-Tc oxide superconductors and related materials. J. Phys.: Condens. Matter, 17 (4), R143.

[7] Booth, J. C., Wu, D. H., Anlage, S. M. (1994). A broadband method for the measurement of the surface impedance of thin films at microwave frequencies. $R e$ view of Scientific Instruments, 65, 2082.

[8] Tosoratti, N., Fastampa, R., Giura, M., Lenzi, V., Sarti, S., Silva, E. (2000). Two techniques for broadband measurement of the surface impedance of high critical temperature superconducting thin films. International Journal of Modern Physics B, 14, 2926.

[9] Silva, E., Pompeo, N., Sarti, S. (2011). Wideband microwave measurements in $\mathrm{Nb} / \mathrm{Pd} 84 \mathrm{Ni} 16 / \mathrm{Nb}$ structures and comparison with thin Nb films. Superconductor Science and Technology, 24, 024018.

[10] Biondi, M. A., Garfunkel, M. P. (1959). Millimeter wave absorption in superconducting aluminum. I. Temperature dependence of the energy gap. Physical Review, $116,853$.

[11] Turner, P. J., Broun, D. M., Kamal, S., Hayden, M. E., Bobowski, J. S., Harris, R., Morga, D. C., Preston, J. S., Bonn, D. A., Hardy, W. N. (2004). Bolometric technique for high-resolution broadband microwave spectroscopy of ultra-low-loss samples. Review of Scientific Instruments, 75, 124.

[12] Nichols, C. S., Shiren, N. S., Laibowitz, R. B., Kazyaka, T. G. (1988). Microwave transmission through films of $\mathrm{YBa}_{2} \mathrm{Cu}_{3} \mathrm{O}_{7-\delta}$. Physical Review B, 38, 11970.

[13] Golosovsky, M., Davidov, D., Rettori, C., Stern, A. (1989). Magnetic field modulation effects on the microwave transmission through superconducting thin films of Y-Ba-Cu-O. Physical Review B, 40, 9299.
[14] Sridhar, S., Kennedy, W. L. (1988). Novel technique to measure the microwave response of high Tc superconductors between 4.2 and 200 K. Review of Scientific Instruments, 59 (4), 531-536.

[15] Silva, E., Lezzerini, A., Lanucara, M., Sarti, S., Marcon, R. (1998). A cavity system for the measurement of the surface resistance at $48 \mathrm{GHz}$ in high-Tc superconductors. Measurement Science and Technology, 9, 275.

[16] Misra, M., Kataria, N. D., Pinto, R., Tonouchi, M., Srivastava, G. P. (2001). Sensitivity of $\mathrm{R}_{s}$-measurement of HTS thin films by three prime resonant techniques: Cavity resonator, dielectric resonator, and microstrip resonator. IEEE Transactions on Applied Superconductivity, 11 (4), 4128-4135.

[17] Krupka, J., Mazierska, J. (2000). Single-crystal dielectric resonators for low-temperature electronics applications. IEEE Transactions on Microwave Theory and Techniques, 48 (7), 1270-1274.

[18] Kim, J., Kim, M. S., Lee, K., Lee, J., Cha, D., Friedman, B. (2003). Development of a near-field scanning microwave microscope using a tunable resonance cavity for high resolution. Measurement Science and Technology, 14 (1), 7-12.

[19] Pompeo, N., Marcon, R., Silva, E. (2007). Dielectric resonators for the measurement of superconductor thin films surface impedance in magnetic fields at high microwave frequencies. Journal of Superconductivity and Novel Magnetism, 20 (1), 71-82.

[20] Krupka, J., Derzakowski, K., Tobar, M., Hartnett, J., Geyer, R. G. (1999). Complex permittivity of some ultralow loss dielectric crystals at cryogenic temperatures. Measurement Science and Technology, 10, 387-392.

[21] Cherpak, N., Barannik, A., Filipov, Yu., Prokopenko, Yu., Vitusevich, S. (2003). Accurate microwave technique of surface resistance measurement of large-area HTS films using sapphire quasi-optical resonator. IEEE Transactions on Applied Superconductivity, 13 (2), 3570-3573.

[22] Barannik, A.A., Bunyaev, S.A., Cherpak, N.T. (2008). On the low-temperature microwave response of a $\mathrm{Y}_{2} \mathrm{Cu}_{3} \mathrm{O}_{7-\delta}$ epitaxial film determined by a new measurement technique. Low Temperature Physics, 34 (12), 977.

[23] Barannik, A., Cherpak, N. T., Tanatar, M. A., Vitusevich, S., Skresanov, V., Canfield, P. C., Prozorov, R. (2013). Millimeter-wave surface impedance of optimally-doped $\mathrm{Ba}\left(\mathrm{Fe}_{1-x} \mathrm{Co}_{x}\right)_{2} \mathrm{As}_{2}$ single crystals. Physical Review B, 87, 014506

[24] Klein, O., Donovan, S., Dressel, M., Grüner, G. (1993). Microwave cavity perturbation technique: Part I: Principles.International Journal of Infrared and Millimeter Waves, 14 (12), 2423-2457.

[25] Donovan, S., Klein, O., Dressel, M., Holczer, K., Grüner, G. (1993). Microwave cavity perturbation tech- 
nique: Part II: Experimental scheme. International Journal of Infrared and Millimeter Waves, 14 (12), 24592487.

[26] Kobayashi, Y., Imai, T., Kayano, H. (1991). Microwave measurement of temperature and current dependences of surface impedance for high- $\mathrm{T}_{c}$ superconductors. IEEE Transactions on Microwave Theory and Techniques, 39 (9), 1530-1538.

[27] Lee, J. H., Yang, W. I., Kim, M. J., Booth, J. C., Leong, K., Schima, S., Rudman, D., Lee, S. Y. (2005). Accurate measurements of the intrinsic surface impedance of thin $\mathrm{YBa}_{2} \mathrm{Cu}_{3} \mathrm{O}_{7-\delta}$ films using a modified two-tone resonator method. IEEE Transactions on Applied Superconductivity, 15 (2), 3700-3705.

[28] Kajfez, D. (1994). Linear fractional curve fitting for measurement of high Q factors. IEEE Transactions on Microwave Theory and Techniques, 42 (7), 1149-1153.

[29] Petersan, P. J., Anlage, S. M. (1998). Measurement of resonant frequency and quality factor of microwave resonators: Comparison of methods. Journal of Applied Physics, 84 (6), 3392-3402.

[30] Press, W. H., Teukolsky, S. A., Vetterling, W. T., Flannery B. P. (2002). Numerical Recipes in C, The Art of Scientific Computing, 2nd Edition. Cambridge University Press.

[31] Hakki, B. W., Coleman, P. D. (1960). A dielectric resonator method of measuring inductive capacities in the millimeter range. IRE Transactions on Microwave Theory and Techniques, 8 (4), 402-410.

[32] Krupka, J., Klinger, M., Kuhn, M., Baranyak, A., Stiller, M., Hinken, J., Modelski, J. (1993). Surface resistance measurements of HTS films by means of sapphire dielectric resonators. IEEE Transactions on Applied Superconductivity, 3 (3), 3043-3048.

[33] Powell, R. L., Fickett, F. R. (1979). Cryogenic Properties of Copper. International Copper Research Association.

[34] Reaney, I. M., Iddles, D. (2006). Microwave dielectric ceramics for resonators and filters in mobile phone networks. Journal of the American Ceramic Society, 89 (7), 2063-2072.

[35] Klein, N., Dähne, U., Schulz, H., Tellmann, N., Kutzner, R., Zaitsev, A. G., Wördenweber, R. (1995). Dielectric properties of rutile and its use in high temperature superconducting resonators. Journal of Applied Physics, 78, 6683.

[36] Tinkham, M. (1996). Introduction to Superconductivity, 2nd Edition. McGraw-Hill.

[37] Blatter, G., Feigel'man, M. V., Geshkenbein, V. B., Larkin, A. I., Vinokur, V. M. (1997). Vortices in high-temperature superconductors. Reviews of Modern Physics, 66, 1377.

[38] Gittleman J., Rosenblum, B. (1966). Radio-frequency resistance in the mixed state for subcritical currents. Physical Review Letters, 16, 734.

[39] Leong, K., Mazierska, J. (2001). Accurate measurements of surface resistance of HTS films using a novel transmission mode Q-Factor technique. Journal of Superconductivity and Novel Magnetism, 41 (1), 93-103.

[40] Leong, K., Mazierska, J. (2002). Precise measurements of the $\mathrm{Q}$ factor of dielectric resonators in the transmission mode-accounting for noise, crosstalk, delay of uncalibrated lines, coupling loss, and coupling reactance. IEEE Transactions on Microwave Theory and Techniques, 50 (9), 2115-2127.

[41] Mazierska, J., Wilker, C. (2001). Accuracy issues in surface resistance measurements of high temperature superconductors using dielectric resonators (corrected). IEEE Transactions on Applied Superconductivity, 11 (4), 4140-4147.

[42] Torokhtii, K., Attanasio, C., Cirillo, C., Ilyina, E.A., Pompeo, N., Sarti, S., Silva, E. (2012). Vortex motion in $\mathrm{Nb} / \mathrm{PdNi} / \mathrm{Nb}$ trilayers: New aspects in the flux flow state. Physica C, 479, 140142.

Submitted July 25, 2013. Accepted May 20, 2014 